\section{SAT0426 SERUM URIC ACID LEVELS PREDICT DAMAGE ACCRUAL IN SYSTEMIC LUPUS ERYTHEMATOSUS PATIENTS}

C. Elera-Fitzcarrald ${ }^{1}, 2$, C. Reategui-Sokolova ${ }^{1}$, R.V. Gamboa-Cárdenas ${ }^{1,3}$, M. Medina ${ }^{1}$, F. Zevallos ${ }^{1}$, V. Pimentel-Quiroz ${ }^{1}$, J.M. Cucho-Venegas ${ }^{1}$, J.L. AlfaroLozano $^{1}$, Z.J. Rodríguez-Bellido ${ }^{1,3}$, C.A. Pastor-Asurza ${ }^{1,3}$, R.A. Perich-Campos ${ }^{1,3}$, G. Alarcón ${ }^{4}$, M.F. Ugarte-Gil ${ }^{1}, 2 .{ }^{1}$ Rheumatology, Hospital Guillermo Almenara Irigoyen, EsSalud; ${ }^{2}$ Universidad Científica del Sur, ${ }^{3}$ Universidad Nacional Mayor de San Marcos, Lima, Peru; ${ }^{4}$ School of Medicine, The University of Alabama at Birmingham, Birmingham, AL, USA

Background: Serum uric acid levels have been reported as predictors of cardiovascular and renal morbidity $\left(,{ }^{1}\right.$ and, of increasing the risk of renal damage in systemic lupus erythematosus (SLE) patients $\left({ }^{2}\right.$ However, their role as predictors of global damage accrual in SLE patients has not been determined.

Objectives: To determine whether uric acid levels predict new damage in SLE patients.

Methods: This is a longitudinal study of SLE patients from a single centre cohort which started in 2012. Visits were performed every six months. Patients with at least two visits were included. Demographic and clinical characteristics as well as treatment were recorded at every visit. Disease activity was ascertained with the Systemic Lupus Erythematosus Disease Activity Index (SLEDAI), and damage with the Systemic Lupus International Collaborating Clinics (SLICC)/American College of Rheumatology (ACR) damage index (SDI). Prednisone use was recorded as current daily dose and time of exposure. Immunosuppressive drugs and antimalarial use was recorded as current, past or never. All variables were ascertained at baseline, with the exception of new damage which was assessed at the subsequent visits. Univariable and multivariable Cox-regression models were performed to determine the impact of uric acid levels on the risk of new damage. Multivariable models were adjusted for age at diagnosis, disease duration, socioeconomic status, SLEDAI, SDI, comorbidities and use of prednisone, immunosuppressive drugs and antimalarials.

Results: Two hundred and thirty-seven patients were evaluated. The mean (SD) age at diagnosis was $35.9(13.1)$ years, 220 patients $(92.8 \%)$ were female, nearly all were Mestizo, disease duration was 7.3 (6.6) years. The mean SLEDAI and SDI were 5.1 (4.2) and 0.9 (1.3), respectively. The Charlson comorbidity index was 0.5 (0.9). Uric acid levels were $4.5(1.4) \mathrm{mg} / \mathrm{dl}$. The mean current prednisone dose $7.1(6.4) \mathrm{mg} / \mathrm{d}$. The duration of exposure to prednisone was $6.9(6.2)$ years. Follow-up time was 3.1 (1.3) years. One hundred and twelve (47.3\%) patients accrued damage during the follow-up. In univariable and multivariable analyses, uric acid levels predicted new damage [HR=1.14 (95\% Cl 1.01-1.28); $\mathrm{p}=0.026$ and $\mathrm{HR}=1-.16(95 \% \mathrm{Cl} 1.00-1.34) ; \mathrm{p}=0.043$, respectively].

Conclusions: Higher uric acid levels predicted the development of new damage in our SLE patients.

\section{REFERENCES:}

[1] Zoccali $C$, et al. Uric acid, hypertension, and cardiovascular and renal complications. Current hypertension reports. 2013;15(6):531-7.

[2] Reategui-Sokolova C, et al. Serum uric acid levels contribute to new renal damage in systemic lupus erythematosus patients. Clinical rheumatology 2017;36(4):845-52.

Disclosure of Interest: None declared

DOI: 10.1136/annrheumdis-2018-eular.6128

\section{SAT0427 PREDICTIVE VALUE OF FETAL UMBILICAL ARTERY DOPPLER IN ADVERSE PREGNANCY OUTCOMES IN PATIENTS WITH LUPUS NEPHRITIS}

D. Chen, Z. Zhan. Rheumatology, The first affiliated hospital of Sun Yat-sen University, guangzhou, China

Background: Compared with the general population, patients with lupus nephritis (LN) are still at high risk of adverse pregnancy outcomes (APOs), including fetal loss, preterm birth and intrauterine growth retardation (IUGR). Umbilical artery is particularly important for placental perfusion and fetal development. Increased umbilical artery resistance could be traced by Doppler velocimetry, which could be used as a screening tool for placenta-related diseases. However, the predictive value in HDP of lupus pregnancies has not been widely assessed.

Objectives: To examine the predictive value of the fetal umbilical artery Doppler on adverse pregnancy outcomes (APOs) in pregnant women with lupus nephritis (LN).
Methods: The clinical data of 158 LN pregnant patients from the First Affiliated Hospital of Sun Yat-sen University were analysed retrospectively.

Results: Totally, 119 were diagnosed before pregnancy and 39 were newly diag nosed during pregnancy. One or more APOs occurred in $74.7 \%$ of patients with $\mathrm{LN}$ and 40 (25.3\%) were without any APOs. Fifty-four of pregnancies (12.2\%) resulted in fetal loss. A total of 55 pregnancies were preterm birth, 24 were IUGR 14 were fetal distress and 9 were neonatal lupus. Doppler pulsatility index (PI), resistance index $(R I)$ as well as $S / D$ value were significantly higher in the APOs groups than in the patients without APOs $(p<0.05)$. The area below the receiver operating characteristic (ROC) curve for $\mathrm{PI}, \mathrm{RI}$ and $\mathrm{S} / \mathrm{D}$ all was $0.7(95 \% \mathrm{C}$ $0.6 \sim 0.8$ ). PI with cut-off value of 0.9 indicated the highest risk of APOs, with sensitivity of $28.3 \%$ and specificity of $97.2 \%$. Regarding 0.6 as the cut-off value for RI to predict APOs, the sensitivity was $45.7 \%$ and the specificity was $80.6 \%$. The optimal cut-off value for S/D was 2.5 , at which sensitivity $(39.1 \%)$ and specificity $(90.0 \%)$ had the best combination.

Conclusions: Pregnancies in LN were more prone to pregnancy loss and preterm birth. Umbilical artery Doppler was a useful monitoring measure for APOs in pregnancies of LN.

Disclosure of Interest: None declared

DOI: 10.1136/annrheumdis-2018-eular.5030

\section{SAT0428 ASSOCIATION BETWEEN ORGAN DAMAGE AND HEALTH-RELATED QUALITY OF LIFE IN SYSTEMIC LUPUS ERYTHEMATOSUS (SLE): A SYSTEMATIC REVIEW}

E.R. Hammond ${ }^{1}$, D.H. Lin ${ }^{2}$, I.B. Murimi ${ }^{2}$, H. Nab ${ }^{3}$, H. Kan ${ }^{2}$, O. Onasanya ${ }^{2}$, J. C. Tierce ${ }^{2}$, X. Wang ${ }^{1}$, B. Desta ${ }^{1}$, G.C. Alexander ${ }^{2}{ }^{1}$ AstraZeneca, Gaithersburg, MD; ${ }^{2}$ Johns Hopkins Bloomberg School of Public Health, Baltimore, MD, USA;

${ }^{3}$ AstraZeneca, Cambridge, UK

Background: Organ damage in SLE is associated with increased morbidity and mortality. The comorbid burden of SLE involves various organ systems and may be associated with pain, fatigue, difficulty with daily activities (including school and work), and emotional well-being, all contributing to high rates of disability. Objectives: We conducted a systematic literature review evaluating association between SLE-related organ damage and health-related quality of life (HRQOL).

Methods: A systematic literature search (January 2000-February 2017) of PubMed, EMBASE, Cochrane Library, and Latin American and Caribbean Health Sciences Literature was conducted to identify studies evaluating association between organ damage (measured by SLICC/ACR Damage Index [SDI]) and HRQOL for adults with SLE. Instruments examined include the Short Form 36 (SF-36: Physical Component Summary [PCS] and Mental Component Summary [MCS], 15 studies); EuroQol 5 Dimensions Questionnaire (EQ-5D) and Fatigue Severity Scale (FSS, 2 studies each); and the LupusQoL, Multidimensional Fatigue Inventory (MFI), Quality of Life Questionnaire of the European Foundation for Osteoporosis (QUALEFFO), and WHO Quality of Life Scale (WHOQOL BREF) (1 study each)

Results: From 10420 articles screened, 20 studies were included (3 prospective cohort studies, 17 cross-sectional studies). The 3 prospective studies evaluated HRQOL by the SF-36, 2 of which modelled SDI as a binary variable. For patients without damage at baseline, any damage accrual over 2 years was associated with a 5.5-fold increased risk of decreased HRQOL compared with those who did not develop new damage (SF-36 overall relative risk [RR] $5.5[95 \% \mathrm{Cl}$ : $1.1-26.6$ $\mathrm{p}=0.03])$. A similar trend toward increased risk of decreased HRQOL was observed with PCS: RR 2.5 (95\% Cl: 0.6-10.0; $\mathrm{p}=0.19)$; and MCS: RR $4.6(95 \%$ $\mathrm{Cl}: 0.9-22.8 ; \mathrm{p}=0.06)$. Any damage at baseline was associated with $67 \%$ decreased odds of achieving minimum clinically important improvement in SF-36 PCS: odds ratio [OR] $0.33(95 \% \mathrm{Cl}: 0.13-0.85 ; \mathrm{p}=0.02)$. However, no association was observed for MCS. When SDI was modelled as a continuous variable, increasing damage was associated dose-dependently with decreased SF-36 PCS compared with SDI=0; SDI 1: -2.64 , $\mathrm{p}<0.001$; SDI 2: $-2.86, \mathrm{p}=0.003$; SDI 3: $-5.94, p<0.001$; SDI $4:-6.35, p<0.001$; and SDI 5+: $-8.11, p<0.001$. In cross-sec tional evaluations, increasing SDI was modestly correlated with decreased HRQOL: SF-36 correlation coefficient score range: -0.12 to -0.30 ; SF-36 regression coefficient score range: -0.011 to -4.65 ; LupusQoL correlation coefficient $0.31, p<0.01$; and WHOQOL-BREF regression coefficient $-0.06, p=0.57$. Increas ing fatigue displayed weak correlation with damage: FSS correlation coefficient range: 0.04 to 0.15 .

Conclusions: Organ damage was associated with decreased HRQOL in most studies evaluated. This review determined heterogeneity in HRQOL instruments and methodology across studies. Improving construct validity of instruments and 